NBER WORKING PAPER SERIES

INEQUALITY WITHIN AND BETWEEN FAMILIES

Eytan Sheshinski

Yoram Weiss

Working Paper No. 405

NATIONAL BUREAU OF ECONOMIC RESEARCH 1050 Massachusetts Avenue

Cambridge MA 02138

November 1979 


\begin{abstract}
Between-family differences in expenditures and output reflect the effect of simultaneous increases in children's ability on the willingness of parents to transfer resources to them. Within-family differences also reflect the attitudes of parents toward disparity among children. In this paper we characterize the conditions on parents' preferences that determine whether between-family differences exceed within-family differences. For an additive utility, within-family differences in expenditures always exceed between-family differences. This may also be true for the max-min utility function if an increase in ability reduces the marginal utility of income. Within-family differences in output (utility or income) can also exceed between-family differences. In this case, the implication for income distribution is that equality is enhanced by a higher correlation of ability between brothers.
\end{abstract}

Professor Eytan Sheshinski Department of Economics Hebrew university Jerusalem, Israel
Professor Yoram Weiss Department of Economics University of Tel Aviv Tel Aviv, Israel 


\section{INEQUALITY WITHIN AND BETWEEN FAMILIES ${ }^{1}$}

$$
\text { by }
$$

\section{Eytan Sheshinski* and Yoram Weiss**}

\section{Introduction}

A major goal of government policy is to affect the income distribution between individuals in society. There are, however, other social institutions which affect the income distribution and their actions may either reinforce or countervail those of the government. The family plays a major role in this respect. The effect of the family on the distribution of income depends on the variation in individual characteristics within and between families and on the rules for allocation of family resources. We are interested in a particular aspect of this problem: the allocation of parental resources among children of different abilities and its effect on the distribution of their future income and welfare.

The nature of the problem can be illustrated by means of a very simple example. Consider two families, each consisting of two children. Let the parents of each family have the same wealth. Children are distinguished solely by their endowed ability. Suppose that half the children's population is of 'high ability' and half of 'low ability.' One possibility is that all differences in ability are within families and hence each family has one 'able' and one 'less able' child. Another possibility is that differences are only between families where one family has two 'able' children and the other has two 'less able' children. We would like to compare the distribution of inputs, e.g., schooling expenditures, and the

$1_{\text {This }}$ research was supported by an ONR contract to the Center for Research on Organizational Efficiency, and by the "Economics of the Family" program at NBER. Any opinions expressed are those of the authors and not those of the National Bureau of Economic Research.

The authors are indebted to Zvi Griliches for suggesting the problem analyzed in this paper.

*The Hebrew Univeristy, Jerusalem.

${ }^{*}$ Te1 Aviv University. 
distribution of outputs, that is, income and utility, in the above two cases.

As noted by Griliches [1978], the relevant question for this comparison is whether a given difference in ability leads to a larger difference in expenditures or income within families than across families. This issue is quite separate from the one analyzed by Becker and Tomes [1976] who inquired whether parents invest more resources in the relatively able child. This latter question is relevant for the comparison of the family's optimal redistributive policy to the hypothetical policy of no intervention.

The purpose of this paper is to characterize the properties of parental preferences that are relevant for the comparison of within- and betweenfamily differences. We limit our discussion to parents' utility functions which are symmetric in children's utility. It is assumed that utilities are comparable within the family. Much of our discussion will focus on two special cases that have received attention in the social choice literature: the additive (utilitarian) and the max-min (utility equalization) functions. Both rules can be justified as egalitarian. The max-min principle is egalitarian ex post, while the utilitarian principle is egalitarian ex ante, equalizing the expected utility of each (unborn) child (see Harsanyi [1975], Myerson [1979] and Appendix). We assume that the utility of each child depends on the transfers he receives from his parents and on his ability. This has to be interpreted as the indirect utility function in which all future individual actions have been 'maximized out.'

Much of the analysis hinges upon the interaction between children's abilities and parents' expenditures in the indirect utility function. This, in turn, depends on the feasible methods of transferring resources 
within the family. If parents can make positive and negative direct transfers that are not conditional on schooling and if ability affects utility only through the production of earnings, then it is possible to separate efficiency from distributive considerations (see Becker and Tomes [1976]). In this case, the differences in schooling expenditures within and between families are identical. In our analysis we assume that such separation is not feasible, either due to the fact that ability may affect utility directly or due to limits on parents' power to enforce negative transfers on their grown-up children. In this sense our analysis is similar to Arrow [1971] and Behrman, Pollack and Taubman [1979] who, however, did not compare within- and between-family differences.

Between-family differences in expenditures and outputs reflect the effect of simultaneous increases in children's ability on the willingness of parents to transfer resources to them. Within-family differences also reflect the attitudes of parents towards disparity among children. In this paper we characterize the conditions on parents' preferences that determine whether between-family differences exceed within-family differences. For an additive utility, within-family differences in expenditures always exceed between-family differences. This may also be true for the max-min utility function if an increase in ability reduces the marginal utility of income. Within-family differences in output (utility or income) can also exceed between-family differences. In this case, the implication for income distribution is that equality is enhanced by a higher correlation of ability between brothers. 


\section{Analysis of Family Allocation Rules}

Consider a family consisting of parents and two children. Denote the consumption of parents by $c$ and the expenditures on each child by $s_{1}$ and $s_{2}$, respectively. The indirect utility function of each child, $u_{i}$, is written $u_{i}=u\left(s_{i}, a_{i}\right), i=1,2$, where $a_{i}$ is an index of child $i$ 's ability and $\frac{\partial u}{\partial s_{i}}>0, \frac{\partial u}{\partial a_{i}}>0$. Parents seek to maxirize a utility function, $V$, which depends on their own consumption and on the utility levels of their children,

$$
\mathrm{V}=\mathrm{V}\left(\mathrm{c}, \mathrm{u}\left(\mathrm{s}_{1}, \mathrm{a}_{1}\right), \mathrm{u}\left(\mathrm{s}_{2}, \mathrm{a}_{2}\right)\right)
$$

where $\mathrm{V}$ is assumed to be strictly monotone, quasi-concave and symmetric in the last two arguments, i.e.,

$$
\mathrm{V}\left(\mathrm{c}, \mathrm{x}_{1}, \mathrm{x}_{2}\right)=\mathrm{V}\left(\mathrm{c}, \mathrm{x}_{2}, \mathrm{x}_{1}\right) \quad \forall \mathrm{c}, \mathrm{x}_{1}, \mathrm{x}_{2} \geq 0
$$

The family's budget constraint is given simply by

$$
c+s_{1}+s_{2}=m
$$

where $\mathrm{m}$ is the family's wealth, to be allocated by the parents. 1

Maximization of (1) subject to (3) yields solutions $\hat{s}_{1}\left(a_{1}, a_{2}\right)$, $\hat{s}_{2}\left(a_{1}, a_{2}\right)$ and $\hat{c}\left(a_{1}, a_{2}\right)$. Wealth will be assumed constant throughout most of the discussion and hence the dependence on $\mathrm{m}$ is suppressed. It is also assumed that these solutions are strictly positive. Our discussion focuses on the following properties of these solutions:

(1) Input and Output Progressivity (Arrow [1971]). The dependence of $\hat{s}_{i}$ and $\hat{u}_{i}=u\left(\hat{s}_{i}\left(a_{1}, a_{i}\right)\right.$ on $a_{i}, i=1,2$; that is, whether the optimum levels of expenditures and utility increase or decrease with own ability; 
(2) Within-Family Differences in Inputs and Outputs (Becker and Tomes [1976]). For $a_{1}>a_{2}$, whether $\hat{s}_{1}\left(a_{1}, a_{2}\right) \geq \hat{s}_{2}\left(a_{1}, a_{2}\right)$ and similarly for utility levels, $\hat{\mathrm{u}}_{1}\left(\mathrm{a}_{1}, \mathrm{a}_{2}\right) \geq \hat{\mathrm{u}}_{2}\left(\mathrm{a}_{1}, \mathrm{a}_{2}\right)$; that is, whether heterogeneous families whose children have different abilities reinforce or compensate for these ability differences;

(3) Between-Family Differences in Inputs and Outputs. For $a_{1}>a_{2}$, whether $\hat{s}_{1}\left(a_{1}, a_{1}\right) \geqslant \hat{s}_{2}\left(a_{2}, a_{2}\right)$ and $\hat{u}_{1}\left(a_{1}, a_{1}\right) \geq \hat{u}_{2}\left(a_{2}, a_{2}\right)$; that is, whether expenditures and utility increase or decrease with ability across homogeneous families whose children's abilities are perfectly correlated.

(4) Comparison of Within- and Between-Family Differences in Inputs and Outputs (Griliches [1978]). For any pair $\left(a_{1}, a_{2}\right)$, whether $\mid \hat{s}_{1}\left(a_{1}, a_{2}\right)-$ $\hat{s}_{2}\left(a_{1}, a_{2}\right) \mid$ is larger or smaller than $\left|\hat{s}_{1}\left(a_{1}, a_{1}\right)-\hat{s}_{2}\left(a_{2}, a_{2}\right)\right|$. Similarly, whether $\left|\hat{u}_{1}\left(a_{1}, a_{2}\right)-\hat{u}_{2}\left(a_{1}, a_{2}\right)\right|$ is larger or smaller than $\mid \hat{u}_{1}\left(a_{1}, a_{1}\right)-$ $\hat{\mathrm{u}}_{2}\left(\mathrm{a}_{2}, \mathrm{a}_{2}\right) \mid$; that is, for given ability differences, whether differences within heterogeneous families are larger or smaller than differences across homogeneous families.

For purposes of exposition we start with a simplified version of the above problem which brings out its essential features. Suppose that ability affects utility only through its effect on earnings capacity and that the production function is multiplicative, i.e., $u_{i}=u\left(s_{i}, a_{i}\right)=$ $f\left(a_{i} s_{i}\right), i=1,2$. Utility is thus identified with (observable) income and, without loss of generality, we can assume that $f\left(a_{i} s_{i}\right)=a_{i} s_{i}$.

The problem can now be reduced to a standard consumer model:

$$
\begin{aligned}
& \operatorname{Max}_{c, u_{1}, u_{2}}\left(c, u_{1}, u_{2}\right) \\
& \text { s.t. } c+p_{1} u_{1}+p_{2} u_{2}=m
\end{aligned}
$$


where $\mathrm{p}_{i}=\frac{1}{\mathrm{a}_{i}}, i=1,2$. With this formulation, the dependence of inputs on own ability is determined by whether demand elasticity is less than or larger than unity in absolute value: $\frac{\partial \hat{s}_{i}}{\partial a_{i}}=-p_{i}^{2} \frac{\partial\left(p_{i} \hat{u}_{i}\right)}{\partial p_{i}}, i=1,2$. The dependence of outputs (i.e., utilities) on own ability is determined by the slope of the uncompensated demand functions: $\frac{\partial \hat{u}_{i}}{\partial a_{i}}=-p_{i}^{2} \frac{\partial \hat{u}_{i}}{\partial p_{i}}, i=1,2$, which is generally assumed to be negative.

The more able child will obtain larger inputs if and only if $1+\frac{\partial \hat{\mathrm{u}}_{i}}{\partial \mathrm{p}_{1}} \frac{\mathrm{p}_{1}}{\hat{\mathrm{u}}_{1}}-\frac{\partial \hat{\mathrm{u}}_{2}}{\partial \mathrm{p}_{1}} \frac{\mathrm{p}_{1}}{\hat{\mathrm{u}}_{2}}>0$, where these are evaluated at $\mathrm{p}_{1}=\mathrm{p}_{2}$. Starting from a state of equality, there are two opposing forces at work. An increase in ability of one child increases his efficiency in producing earnings. On the other hand, since his utility is higher, it reduces the relative weight given to him in the parents' utility. The outcome is therefore ambiguous (see Becker and Tomes [1976]). In contrast, there is no ambiguity with respect to within-family differences in utility. 
Proposition 1. $\quad \hat{\mathrm{u}}_{1} \geq \hat{\mathrm{u}}_{2}$ as $\mathrm{a}_{1} \geq \mathrm{a}_{2}$.

Proof. Let $a_{1}>a_{2}$, i.e., $p_{1}<p_{2}$, and suppose that $\hat{u}_{1}\left(a_{1}, a_{2}\right)<\hat{u}_{2}\left(a_{1}, a_{2}\right)$. Then $\mathrm{p}_{1} \hat{\mathrm{u}}_{1}+\mathrm{p}_{2} \hat{\mathrm{u}}_{2}>\mathrm{p}_{1} \hat{\mathrm{u}}_{2}+\mathrm{p}_{2} \hat{\mathrm{u}}_{1}$. However, by symmetry, $\mathrm{V}\left(\mathrm{c}, \hat{\mathrm{u}}_{1}, \hat{\mathrm{u}}_{2}\right)=$ $\mathrm{V}\left(\mathrm{c}, \hat{\mathrm{u}}_{2}, \hat{\mathrm{u}}_{1}\right)$, for any c. By strict quasi-concavity, there exists therefore a feasible allocation that yields a higher $V$. Hence, $\left(\hat{u}_{1}, \hat{u}_{2}\right)$ cannot be optima1 \|.

In families with identical children $p_{1}=p_{2}$ and we can define a composite good $\mathrm{p}_{1}^{0}\left(\mathrm{u}_{1}+\mathrm{u}_{2}\right)$, where $\mathrm{p}_{1}^{0}$ is some base level of ability. Between-family differences in expenditures depend on the uncompensated cross elasticity of parents' consumption with respect to this composite good.

Proposition 2. If $\hat{\mathrm{c}}$ is a gross-substitute (complement) to $\hat{\mathrm{u}}_{1}+\hat{\mathrm{u}}_{2}$ then, among homogeneous families, expenditures increase (decrease) with the children's ability.

Proof. Since $V$ is symmetric in $u_{1}$ and $u_{2}, \hat{s}_{1}(a, a)=\hat{s}_{2}(a, a)=$ $\frac{1}{2}[m-\hat{c}(a, a)]$, for any $a$. Thus, $\frac{d \hat{s}_{1}(a, a)}{d a}=\frac{d \hat{s}_{2}(a, a)}{d a}=-\frac{1}{2} \frac{d \hat{c}(a, a)}{d a} \|$.

Increased ability of children has two opposing effects on parents' consumption: an income effect which is 1ikely to induce higher consumption by parents and a substitution effect which encourages an increase in expenditures on children and a reduction in parents' consumption.

We are now ready to compare within- and between-family differences in inputs and outputs. 
Proposition 3 . If $\hat{\mathrm{c}}$, $\hat{\mathrm{u}}_{1}$ and $\hat{\mathrm{u}}_{2}$ are all either gross-substitutes or grosscomplements, thenwithin-family differences exceed between-family differences in expenditures. Specifically, for $a_{1}>a_{2}$,

$$
\hat{s}_{1}\left(a_{1}, a_{2}\right)>\hat{s}_{1}\left(a_{1}, a_{1}\right)>\hat{s}_{2}\left(a_{2}, a_{2}\right)>\hat{s}_{2}\left(a_{1}, a_{2}\right)
$$

When the goods are gross-substitutes, all inequalities are reversed in the case of gross-complements.

Proof. The middle inequality follows from Proposition 2. The other two inequalities follow by assumption: $\frac{\partial \hat{u}_{1}}{\partial \mathrm{p}_{2}}>0$ implies that when $a_{1}>a_{2}$, $\hat{\mathrm{u}}_{1}\left(\mathrm{a}_{1}, \mathrm{a}_{2}\right)>\hat{\mathrm{u}}_{1}\left(\mathrm{a}_{1}, \mathrm{a}_{1}\right)$. But $\hat{\mathrm{u}}_{1}\left(\mathrm{a}_{1}, \mathrm{a}_{2}\right)=\mathrm{a}_{1} \hat{\mathrm{s}}_{1}\left(\mathrm{a}_{1}, \mathrm{a}_{2}\right)$ and $\hat{\mathrm{u}}_{1}\left(\mathrm{a}_{1}, \mathrm{a}_{1}\right)=$ $a_{1} \hat{s}_{1}\left(a_{1}, a_{1}\right)$. Hence $\hat{s}_{1}\left(a_{1}, a_{2}\right)>\hat{s}_{1}\left(a_{1}, a_{1}\right) \|$.

Proposition 4. If $\hat{\mathrm{c}}, \hat{\mathrm{u}}_{1}$ and $\hat{\mathrm{u}}_{2}$ are gross-substitutes then within-family differences exceed between-family differences in utility. Specifically, for $a_{1}>a_{2}$,

$$
\hat{\mathrm{u}}_{1}\left(\mathrm{a}_{1}, \mathrm{a}_{2}\right)>\hat{\mathrm{u}}_{1}\left(\mathrm{a}_{1}, \mathrm{a}_{1}\right)>\hat{\mathrm{u}}_{2}\left(\mathrm{a}_{2}, \mathrm{a}_{2}\right)>\hat{\mathrm{u}}_{2}\left(\mathrm{a}_{1}, \mathrm{a}_{2}\right)
$$

Proof. The middle inequality follows immediately from Proposition 3 since the allocation of expenditures within the family reinforces ability differences. The other inequalities follow directly from the assumption \|.

Contrary to the previous case, gross-complementarity does not imply the same result. Indeed, we have the following.

Proposition 5. If $\hat{\mathrm{u}}_{1}$ and $\hat{\mathrm{u}}_{2}$ are gross-complements, then between-family differences exceed within-family differences in utility. Specifically, for $a_{1}>a_{2}$, 


$$
\hat{\mathrm{u}}_{1}\left(\mathrm{a}_{1}, \mathrm{a}_{1}\right)>\hat{\mathrm{u}}_{1}\left(\mathrm{a}_{1}, \mathrm{a}_{2}\right)>\hat{\mathrm{u}}_{2}\left(\mathrm{a}_{1}, \mathrm{a}_{2}\right)>\hat{\mathrm{u}}_{2}\left(\mathrm{a}_{2}, \mathrm{a}_{2}\right) \text {. }
$$

Proof. The middle inequality follows from Proposition 1. The other inequalities follow directly from the assumptions $\|$.

The above propositions can be used to determine the implications of alternative specifications of the family utility function for the allocation of family resources.

We shall examine these implications for the ex ante (additive utility) and for the ex post (max-min) egalitarian criteria.

\section{A. Additive Utility}

Let $\mathrm{V}$ have the form

$$
v\left(c, u\left(s_{1}, a_{1}\right), u\left(s_{2}, a_{2}\right)\right)=w(c)+v\left(u_{1}\right)+v\left(u_{2}\right)
$$

where $u_{i}=a_{i} s_{i}, i=1,2$, and $w$ and $v$ are monotone, strictly concave functions. In this case, the conditions of Propositions 1-5 depend solely on the signs of $\frac{\partial^{2} v}{\partial a_{i} \partial s_{i}}=v^{\prime}\left(u_{i}\right)+u_{i} v^{\prime \prime}\left(u_{i}\right), i=1,2$. If the marginal utility of expenditures increases with ability for both $\hat{s}_{1}$ and $\hat{s}_{2}$ then $\hat{u}_{1}$, $\hat{u}_{2}$ and $\hat{c}$ will be gross-substitutes. In the opposite case all goods are grosscomplements. Hence, for an additive utility function, within-family differences always exceed between-family differences in expenditures.

On the other hand, within-family differences exceed or are less than between-family differences in utility according as the marginal utility of expenditures increases or decreases with ability. 


\section{B. Max-min}

Let $\mathrm{V}$ have the form

$$
v\left(c, u\left(s, a_{1}\right), u\left(s_{2}, a_{2}\right)\right)=w(c)+\min \left[v\left(u_{1}\right), v\left(u_{2}\right)\right]
$$

where $u_{i}=a_{i} s_{i}, i=1,2$. Clearly, $\hat{u}_{1}=\hat{u}_{2}$ and hence these are grosscomplements. Thus, only Propositions 2, 3 and 5 are potentially applicable. As in the previous case, it can be shown that $\hat{c}$ is a gross-complement to $\hat{u}_{1}$ (and $\hat{u}_{2}$ ) if and only if $v^{\prime}\left(u_{i}\right)+u_{i} v^{\prime \prime}\left(u_{i}\right)<0, i=1,2$.

In this case the max-min function too entails that within-family differences exceed between-family differences in expenditures. Since the conditions of Proposition 5 hold, between-family differences exceed within-family differences in utility. In particular, therefore, the max-min criterion implies that an able child enjoys a lower utility level in a heterogeneous family than in a homogeneous family. This may raise a question with regard to the 'fairness' of the max-min criterion in the present context. That is, if children or parents compare utilities across families, then within-family equalization may be judged unfair towards the able child. The ex ante egalitarian (sum of utilities) rule bypasses this difficulty since it accounts for the chance of the able child to be born into a family with another able child. ${ }^{2}$

As the above two examples illustrate, within-family differences may well exceed between-family differences in expenditures. Variations in children's ability induces two kinds of transfers from parents to children and among children. These may reinforce or offset each other. In the additive case the same cause that leads parents to transfer resources to the able (or less able) child, namely, his higher marginal utility, also induces them to transfer resources from his brother if he is of different 
ability. In the max-min case parents may also transfer resources to their less able child if they perceive his marginal utility to be higher. If children within the family have different abilities, parents will also transfer resources from the able to the less able, thereby reinforcing between-family differences. In the opposite case, however, when the marginal utility of the able child is higher, then in homogeneous families parents will be willing to transfer resources to them, but in heterogeneous families parents will tax the able child in favor of the less able. Between- and within-family differences in expenditures will therefore be of opposite sign.

Consider now the more general case of nonlinear 'production functions' $u_{i}=u\left(s_{i}, a_{i}\right), i=1,2$. Since $u$ is strictly monotone in $s$, one can invert $u$ to obtain $s_{i}=e\left(u_{i}, a_{i}\right), i=1,2$. With this transformation the maximization problem is

$$
\begin{aligned}
& \operatorname{Max}_{c, u_{1}, u_{2}} v\left(c, u_{1}, u_{2}\right) \\
& \text { s.t. } c+p_{1} u_{1}+p_{2} u_{2}=m
\end{aligned}
$$

where $p_{i}\left(u_{i}, a_{i}\right)=\frac{e\left(u_{i}, a_{i}\right)}{u_{i}}, i=1,2$. Previously we have analyzed the case in which $e_{i}$ is linear in $u_{i}$ and hence $p_{i}$ is independent of $u_{i}$, giving rise to a standard consumer problem. In the present case, Propositions 1-5 have to be reinterpreted as describing outcomes of shifts in the 'supply functions,' $p_{i}\left(u_{i}, a_{i}\right)$, due to changes in $a_{i}$. For instance, $\hat{u}_{1}$ and $\hat{u}_{2}$ are gross-substitutes when $\frac{\partial \hat{u}_{i}}{\partial a_{j}}<0, i \neq j$. However, such relations now depend jointly on utility, V, and on the 'production function,' u. 


\section{Unobservable Utilities}

In the previous section we have assumed that the utility of children can be directly observed and have placed restrictions on parents' preferences. When children's utilities are unobservable one cannot separately identify the functional forms of parents' and children's utilities. Meaningful restrictions must therefore be in terms of expenditure allocation patterns. As before, we shall investigate two cases, the additive and the max-min utility functions.

\section{A. Additive Utility}

Let

$$
v\left(c, u\left(s_{1}, a_{1}\right), u\left(s_{2}, a_{2}\right)\right)=w(c)+v\left(s_{1}, a_{1}\right)+v\left(s_{2}, a_{2}\right)
$$

where $w$ and $v$ are strictly concave with respect to $c$ and $s_{i}$.

The crucial restriction to this specification is that the marginal rate of substitution between parents' consumption and expenditures on child 1 are independent of the ability of child $j, j \neq i$.

A very simple geometrical argument can be used to show that the results in the previous section pertaining to the additive specification carry over to the present, more general case. Specifically, within-family differences always exceed between-family differences in expenditures.

In Figure 1, we depict the solutions for each of the first-order conditions (F.O.C.) of the problem:

$$
\begin{aligned}
& \frac{\partial V}{\partial s_{2}}=-w^{\prime}\left(m-s_{1}-s_{2}\right)+v_{1}\left(s_{1}, a_{1}\right)=0 \\
& \frac{\partial V}{\partial s_{2}}=-w^{\prime}\left(m-s_{1}-s_{2}\right)+v_{1}\left(s_{2}, a_{2}\right)=0
\end{aligned}
$$

where $v_{1}=\partial v / \partial s$ 


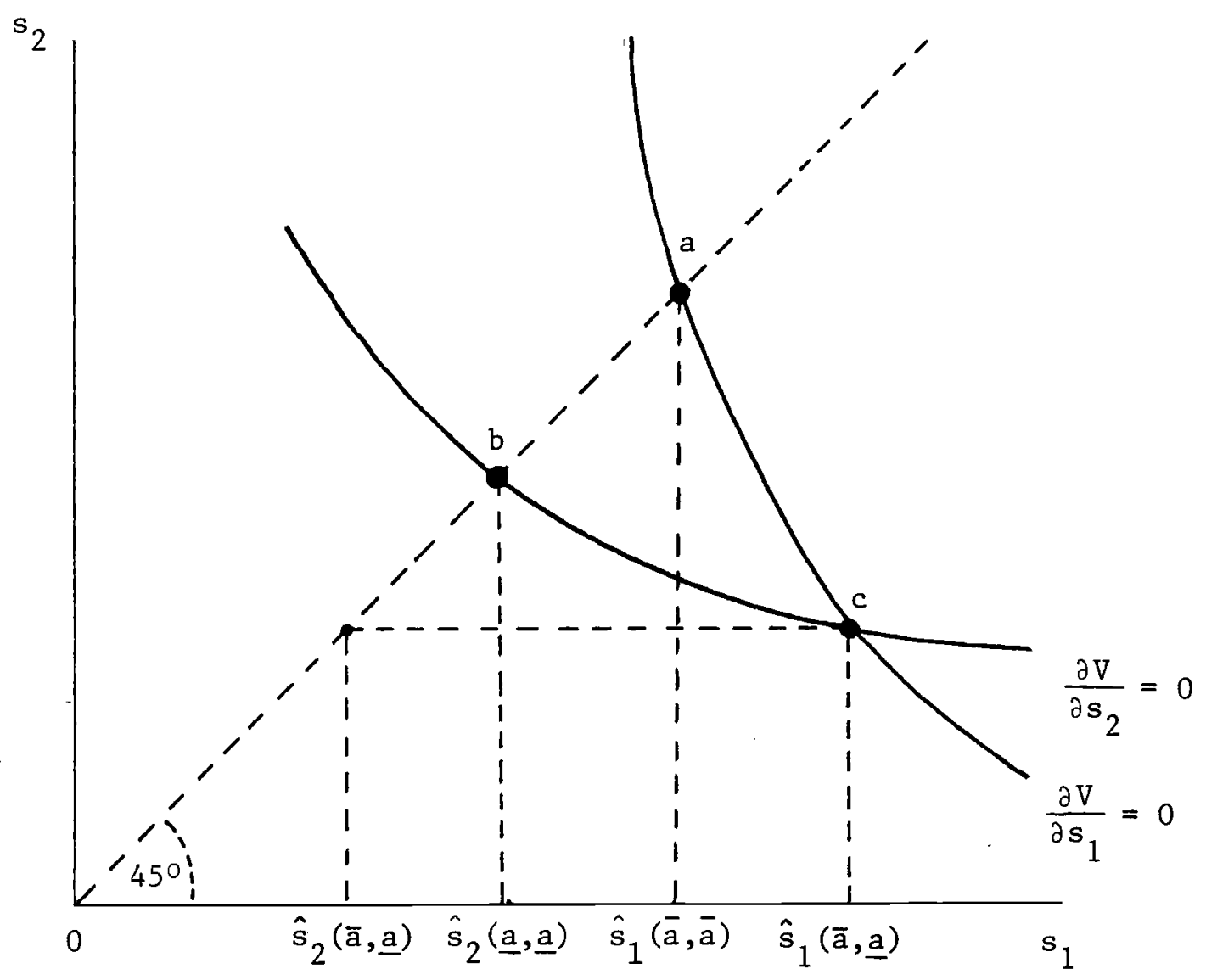

Figure 1

Strict concavity implies that the curves are negatively sloped and intersect at point $c\left(\hat{s}_{1}(\bar{a}, \underline{a}), \hat{s}_{2}(\bar{a}, \underline{a})\right)$. Consider initially the allocation when the two children are of equal ability, say, $a_{1}=a_{2}=\bar{a}$. Due to symmetry the two curves must intersect on the $45^{\circ}$ line at point a. Suppose now that the ability of the second child is reduced to $a_{2}=\underline{a}<\bar{a}$. Due to the additive specification, only the curve $\frac{\partial V}{\partial s_{2}}=0$ shifts. Suppose that $v_{12}(s, a)>0$. Then the solution corresponding to this variation must be below the $45^{\circ}$ line, that is, at point $c$, expenditures on the able exceed those on the less able child. Furthermore, the solution for the case $a_{1}=a_{2}=\underline{a}$ is at point $b$, so as seen, 


$$
s_{1}(\bar{a}, \underline{a})>s_{1}(\bar{a}, \bar{a})>s_{2}(\underline{a}, \underline{a})>s_{2}(\bar{a}, \underline{a})
$$

This chain is reversed when $v_{12}(s, a)<0$. Thus, in this case too, withinfamily differences exceed between-family differences.

\section{B. Max-min}

Let

$$
v\left(c, u\left(s_{1}, a_{1}\right), u\left(s_{2}, a_{2}\right)\right)=w(c)+\min \left[v\left(s_{1}, a_{1}\right), v\left(s_{2}, a_{2}\right)\right]
$$

Assuming, as we have throughout, that the solution is strictly positive, maximization of (13) is equivalent to the maximization of (11) subject to the constraint

$$
u\left(s_{1}, a_{1}\right)-u\left(s_{2}, a_{2}\right)=0
$$

The F.o.C. can be written

$$
\begin{aligned}
& \frac{\partial V}{\partial s_{1}}=-w^{\prime}\left(m-s_{1}-s_{2}\right)+(1+\lambda) v_{1}\left(s_{1}, a_{1}\right)=0 \\
& \frac{\partial V}{\partial s_{2}}=-w^{\prime}\left(m-s_{1}-s_{2}\right)+(1-\lambda) v_{1}\left(s_{2}, a_{2}\right)=0
\end{aligned}
$$

where $-1<\lambda<1$ is the Lagrange multiplier associated with (14). We may use the same diagram as before to analyze the solution. Suppose initially that $a_{1}=a_{2}=\bar{a}$. Then (14) is described by the $45^{\circ}$ line and the solution is $\hat{s}_{1}(a, a)=\hat{s}_{2}(a, a)$. Now decrease $a_{2}$ to $\underline{a}<\bar{a}$.

Suppose that $v_{12}(s, a)<0$. The curve $-w^{\prime}+v_{1}\left(s_{2}, a_{2}\right)=0$ shifts upwards as described and intersects the curve $-w^{\prime}+v_{1}\left(s_{1}, \bar{a}\right)=0$ at point $b$. The curve that represents contraint (14) also shifts upwards. It can be shown that if 


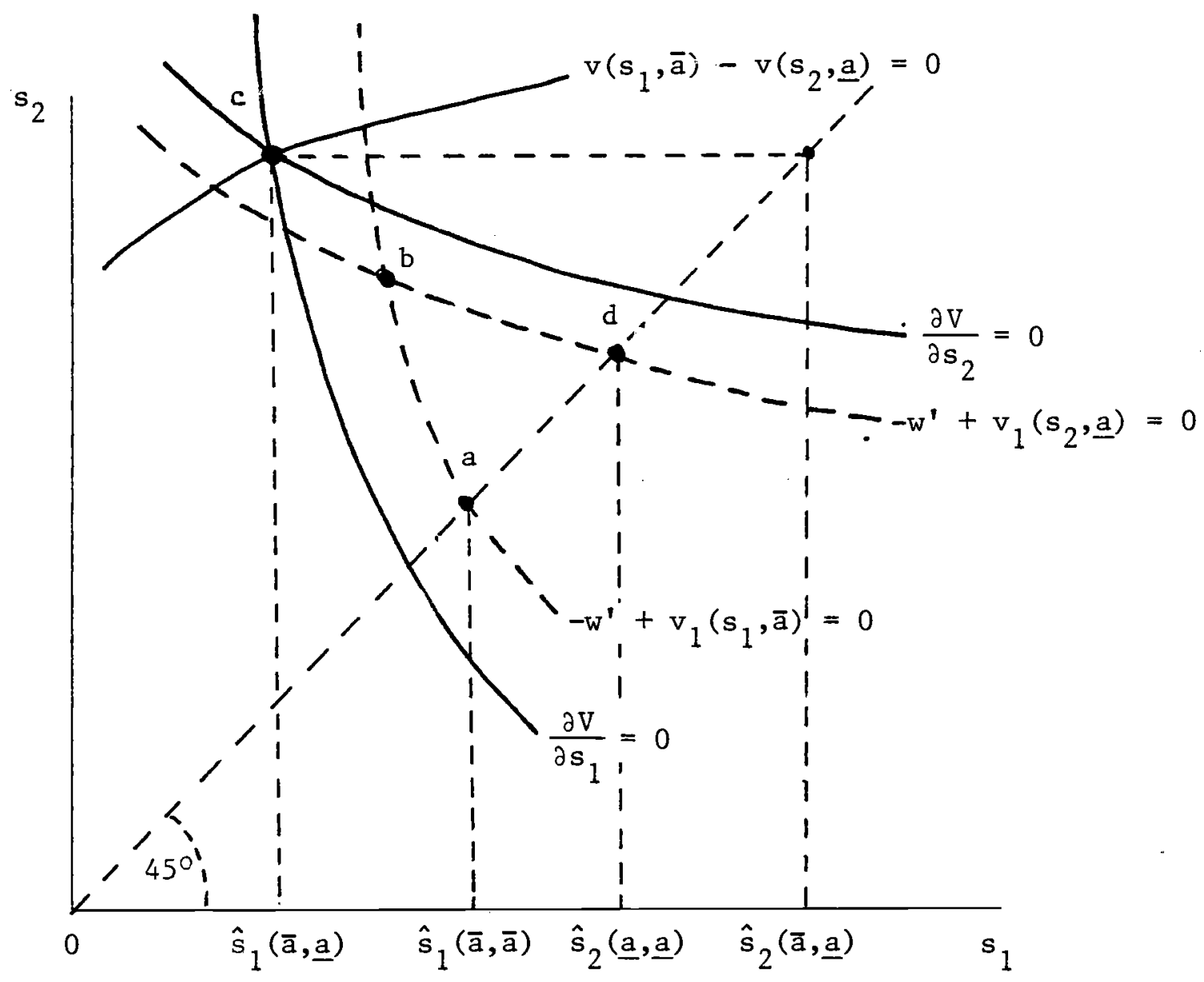

Figure 2

$$
v_{12}(s, a)>\frac{v_{2}(s, a)}{v_{1}(s, a)} v_{11}(s, a)
$$

for all $(s, a)$, which is the standard normality assumption for a, then $v\left(s_{1}, \bar{a}\right)>v\left(s_{2}, \underline{a}\right)$ at point $b$. Hence $\lambda>0$ and the curves $\frac{\partial V}{\partial s_{1}}=0$ and $\frac{\partial \mathrm{V}}{\partial \mathrm{s}_{2}}=0$ intersect at point $c$, yielding the solution $\hat{\mathrm{s}}_{1}(\overline{\mathrm{a}}, \underline{\mathrm{a}})$ and $\hat{\mathrm{s}}_{2}(\overline{\mathrm{a}}, \underline{\mathrm{a}})$. We have thus demonstrated the following:

Proposition 6. If (16) holds for any $s$ and $a$ and if $v_{12}<0$, then withinfamily differences exceed between-family differences in expenditures. 
Proof. Differentiating the F.O.C. (15) it can be shown that the conditions in the Proposition guarantee, respectively, that $\frac{\mathrm{d} \hat{s}_{i}(a, a)}{d a}<0$ and
$\partial \hat{s}_{j}\left(a_{i}, a_{j}\right)$ $\frac{\partial \hat{s}_{i}\left(a_{i}, a_{j}\right)}{\partial a_{j}}>0, i \neq j, i, j=1,2 \|$.

\section{Wealth Effects}

The attitude of families towards inequality among children depends, in general, on the family's wealth. A natural question is whether inequality increases or decreases with the level of wealth. This can be easily analyzed within the framework of Section 2. For the additive case, attitudes towards inequality in output are summarized by the index $-\frac{v^{\prime \prime}(u)}{v^{\prime}(u)}$. Specifically, from the F.O.C., when $u_{1}>u_{2}$ (i.e., $a_{1}>a_{2}$ ),

$$
\operatorname{sgn} \frac{d\left(\hat{u}_{1}-\hat{u}_{2}\right)}{d m}=\operatorname{sgn} \frac{d}{d u}\left[\frac{v^{\prime \prime}(u)}{v^{\prime}(u)}\right]
$$

Although for choices under risk the index of absolute risk-aversion, $-\frac{v^{\prime \prime}}{v^{\prime}}$, is generally assumed to be decreasing in $u$, it is perhaps more plausible to assume the opposite when the degree of inequality within families is concerned.

The inequality in expenditures is related to family preferences by

$$
\operatorname{sgn} \frac{d\left(\hat{s}_{1}-\hat{s}_{2}\right)}{d m}=\operatorname{sgn} \frac{d}{d u}\left[\frac{v^{\prime \prime}(u)}{v^{\prime}(u)^{2}}\right]
$$

for $\hat{u}_{1}>\hat{u}_{2}$ (i.e., $a_{1}>a_{2}$ ). The condition that $-\frac{v^{\prime \prime}}{v^{\prime}}$ decreases in $u$ is clearly sufficient for $\frac{d\left(\hat{s}_{1}-\hat{s}_{2}\right)}{d m}>0$. This may imply, however, an increase or a decrease in inequality depending on the sign of $\hat{s}_{1}-\hat{s}_{2}$. Note further that if $-\frac{v^{\prime \prime}}{v^{\prime}}$ is increasing in $u$, that is, the family prefers higher equality in outputs as wealth increases, the inequality in expenditures may increase with wealth. 
Under the max-min criterion full equality in outputs is attained at all wealth levels. In terms of expenditures, however,

$$
\frac{d\left(\hat{s}_{1}-\hat{s}_{2}\right)}{d m}=\left(\frac{1}{a_{1}}-\frac{1}{a_{2}}\right) \frac{d \hat{u}_{1}}{d m}
$$

For $a_{1}>a_{2}$, and hence $\hat{s}_{1}<\hat{s}_{2}$, this implies that inequality increases with wealth.

\section{Efficient Allocations}

Throughout the discussion we have assumed that parents' transfers to children interact with ability in the indirect utility function. Typically, ability interacts with schooling expenditures in the production of earnings. However, if there are other modes of direct wealth transfers then at the optimum, unless ability affects utility directly, the level of utility is independent of ability. Suppose that the production function of earnings, $y$, is given by $u=f(s, a)$. The indirect utility function, $u$, is $u(x+y)$, where $\mathrm{x}$ is direct wealth transfers from parents. The family's problem is restated as

$$
\begin{aligned}
& \underset{\operatorname{Max}}{\operatorname{Max}} \mathrm{V}\left(\mathrm{c}, \mathrm{u}\left(\mathrm{f}\left(\mathrm{s}_{1}, \mathrm{a}_{1}\right)+\mathrm{x}_{1}\right), \mathrm{u}\left(\mathrm{f}\left(\mathrm{s}_{2}, \mathrm{a}_{2}\right)+\mathrm{x}_{2}\right)\right) \\
& \text { s.t. } \mathrm{c}+\mathrm{x}_{1}, \mathrm{x}_{2}+\mathrm{s}_{2}+\mathrm{x}_{1}+\mathrm{x}_{2}=\mathrm{m}
\end{aligned}
$$

If negative $x_{i}$ are admissible then, at the optimum, utility of children is fully equalized and the allocation $\hat{s}_{1}$ and $\hat{s}_{2}$ satisfies $f_{1}\left(\hat{s}_{1}, a_{i}\right)=1$, $i=1,2$. We shall refer to this as the efficient solution. In contrast to the previous discussion, the allocation of $\hat{s}_{i}$ to each child depends only on his own ability. Thus, between- and within-family differences coincide. 
The efficient solution will also be attained when schooling decisions are made outside the family, either by individuals with access to the capital market or by a planner who can enforce income transfers across individuals.

In the absence of direct income transfers, the family's allocation of expenditures will generally not be efficient. ${ }^{3}$ Distributive considerations will also play a role since the allocation to each child depends on family income and on his brother's ability. It is impossible to compare the second-best allocation to the efficient one. If family's wealth happens to be at the level that would yield the same total expenditure on children as in the efficient allocation, then the distribution of expenditure within the family will be less favorable to the able one than the efficient allocation. 4 At other levels of family income the two allocation rules are not comparable.

\section{Implications for the Distribution of Income}

The implications of our analysis for the distribution of income can be described simply in the framework of the model presented in Section 2 . Suppose that the earnings function, $y$, is of the form:

$$
y=(a s)^{\gamma}
$$

and that the family allocation rule is approximated by:

$$
\left(\begin{array}{cc}
\log s_{1} \\
\log s_{2}
\end{array}\right)=\left(\begin{array}{ll}
\alpha_{1} & \alpha_{2} \\
\alpha_{2} & \alpha_{1}
\end{array}\right)\left(\begin{array}{ll}
\log a_{1} \\
\log a_{2}
\end{array}\right)
$$

Due to Proposition $1,1+\alpha_{1}, \alpha_{2}>0$. Denoting by $\sigma_{x}^{2}$ the variance of $\log x$, we can write the variance of the $\log$ of children's earnings as 


$$
\sigma_{\mathrm{y}}^{2}=\gamma_{\mathrm{a}}^{2} \sigma^{2}\left(\left(1+\alpha_{1}\right)^{2}+\alpha_{2}^{2}+2 \rho \alpha_{2}\left(1+\alpha_{1}\right)\right)
$$

where $\mathrm{p}$ is the correlation in ability between brothers. Assuming that $\mathrm{y}_{i}$ has a negatively sloped demand, $1+\alpha_{i}>0, i=1,2$. Suppose that $\alpha_{2}<0$. This corresponds to the case in which the two children can be viewed as gross-substitutes. In the case of no systematic differences in ability between families $\rho$ will be zero, while if $\rho=1$ all differences in ability are between families. The implication of Proposition 2 is that income inequality will be larger in the former case.

The interaction of a positive correlation between children' ability and negative within-family cross effects reduces the inequality in earnings relative to the case of random assignments of children to families. Since within-family differences in income exceed betweenfamily differences, income equality is enhanced by a more egalitarian distribution of ability in the family. The converse holds when $\alpha_{2}>0$, i.e., when children are gross-complements.

In the extreme case of only between-family differences, i.e., $\rho=1$, the family affects the income distribution only through transfers from parents to children. If it is assumed that parents' consumption is a gross-substitute to children's utility (income), i.e., $\alpha_{1}+\alpha_{2}>0$, then the family can be viewed as contributing to inequality relative to the case of no intervention, i.e., $\alpha_{1}=\alpha_{2}=0$. If the correlation between children's ability is less than unity, then the family will have an additional effect on inequality due to transfers of earnings capacity among children. This contribution to income inequality will be positive if $\alpha_{2}>0$, and negative if $\alpha_{2}<0$. 
The analysis can be extended to incorporate variations in family wealth. As explained in Section 4, family wealth is likely to interact with ability in the family allocation rule. Furthermore, it is probable that children's ability and parents' income are correlated. In principle, one can derive from these relations the intergenerational laws of motion for the income distribution (see Becker and Tomes [1979]), but we sha11 not pursue this issue here. 


\section{Footnotes}

1. Future earnings of children are not subject to redistribution and are reflected in the indirect utility of each child. For a case in which the allocation scheme also affects the size of wealth to be distributed, see Green and Sheshinski [1975].

2. One may view such ex ante evaluation as irrelevant, insisting on ex post fairness and yet be satisfied with a weaker form of within-family equalization. One plausible rule is to maximize the sum of children's utilities subject to the constraint that neither receives less than he would have received if all children had his ability.

3. In some special cases, the efficient solution is attained even in the absence of direct transfers. In particular, if $v_{1}(c, u, u)=v_{2}(c, u, u)=$ $\mathrm{V}_{3}(\mathrm{c}, \mathrm{u}, \mathrm{u})$ for any $\mathrm{c}$ and $\mathrm{u}\left(\mathrm{e} . \mathrm{g} ., \mathrm{V}\left(\mathrm{c}, \mathrm{u}_{1}, \mathrm{u}_{2}\right)=\mathrm{F}\left(\mathrm{c}+\mathrm{u}_{1}+\mathrm{u}_{2}\right)+\mathrm{G}\left(\mathrm{u}_{1}-\mathrm{u}_{2}\right)^{2}\right)$, then in families with identical children the allocation will be efficient. In this case, between-family differences are identical to the differences that exist when income transfers are feasible. However, within-family differences are not efficient.

4. The within-family allocation satisfies

$$
\frac{f_{1}\left(\hat{s}_{1}, a_{1}\right)}{f_{2}\left(\hat{s}_{2}, a_{2}\right)}=\frac{v_{2}\left(u_{1}, u_{2}\right)}{v_{1}\left(u_{1}, u_{2}\right)}
$$

Assume $a_{1}>a_{2}$; then due to symmetry and quasi-concavity of $\mathrm{V}, \mathrm{u}_{1}>\mathrm{u}_{2}$ and $\mathrm{v}_{1}\left(\mathrm{u}_{1}, \mathrm{u}_{2}\right)<\mathrm{v}_{2}\left(\mathrm{u}_{1}, \mathrm{u}_{2}\right)$ at the optimum. Thus, the ratio on the R.H.S. is larger than 1 . The efficient allocation satisfies

$$
\frac{\mathrm{f}_{1}\left(s_{1}^{*}, \mathrm{a}_{1}\right)}{\mathrm{f}_{1}\left(\mathrm{~s}_{2}^{\star}, \mathrm{a}_{2}\right)}=1
$$

By assumption, $s_{1}^{*}+s_{2}^{*}=\hat{s}_{1}+\hat{s}_{2}$ and $f_{11}<0$. It must therefore be the case that $\hat{s}_{1}<s_{1}^{*}, \hat{s}_{2}>s_{2}^{*}$. This is the comparison made by Behrman, Pollak and Taubman [1979] who assume that total expenditures are independent of children's ability and, accordingly, test for the concavity of $V$. They find that parents' utility is concave in children's incomes and that the allocation of expenditures is therefore inefficient. In general, however, total expenditures will depend on children's ability, giving rise to between-family differences. 


\section{$\underline{\text { References }}$}

Arrow, K. J. [1971], "A Utilitarian Approach to the Concept of Equality in Public Expenditures," Quarterly Journal of Economics, 85, 409-415.

Becker, G. S. and N. Tomes [1976], "Child Endowments and the Quantity and Quality of Children," Journal of Political Economy, 84, 143-162.

Becker, G. S. and N. Tomes [1979], "A Theory of Distribution of Income and Intergenerational Mobility," forthcoming in Journal of Political Economy.

Behrman, J., R. A. Pollack and P. Taubman [1979], "Parental Preferences and Parental Investments in Their Progeny," University of Pennsylvania, unpublished.

Green, J. R. and E. Sheshinski [1976], "A Note on the Progressivity of Public Expenditures," Quarterly Journal of Economics, 89, 138-144.

Griliches, Z. [1978], "Sibling Models and Data in Economics: Beginnings of a Survey," to appear in the Journal of Political Economy.

Harsanyi, J. C. [1975], "Nonlinear Social Welfare Functions," Theory and Decision, 6, 311-332.

Myerson, R. B. [1979], "Interpersonal Comparison of Utility and the Timing Effect in Social Choice Problems," Discussion Paper No. 321, Center for Mathematical Studies in Economics and Management, Northwestern University. 


\section{Appendix}

The purpose of this appendix is to prove the equivalence of ex ante egalitarianism and ex post utilitarianism in the context of redistribution within the family.

A family consists of parents (viewed as a single unit) and $k$ children, denoted by $i, i=1,2, \cdots, k$. Let $a_{i}$ denote the ability of child $i$ and $a=\left(a_{1}, a_{2}, \cdots, a_{k}\right)$. It is assumed that a is a random vector with probability distribution $F(a)$. Let $s_{i}(a)$ be the allocation to child $i$ given a, $s(a)=\left(s_{1}(a), s_{2}(a), \cdots, s_{k}(a)\right)$, and $m-\sum_{i=1}^{k} s_{i}(a)$ the allocation to parents (m-family income, constant). Let $u=u\left(s_{i}(a), a_{i}\right)$ be the utility of child $i$ given $a, u_{1}=\left(\partial / \partial s_{i}\right) u\left(s_{i}(a), a_{i}\right)>0$ and $u_{11}<0$. Let $w=w\left(m-\sum_{i=1}^{k} s_{i}\right)$ be the parents' utility, $w^{\prime}>0, w^{\prime \prime}<0$.

Consider the following two problems.

\section{A. Ex Ante Egalitarianism}

(A.1) $\underset{s(a)}{\operatorname{Max}} E\left[u\left(s_{1}(a), a_{1}\right)+m\left(m-\sum_{i=1}^{k} s_{i}(a)\right)\right]$

subject to

$$
E\left[u\left(s_{i}(a), a_{i}\right)-u\left(s_{1}(a), a_{1}\right)\right]=0, i=2,3, \cdots, k
$$

where

$$
E[x(a)]=\iint \ldots \int x(a) d F(a)
$$

Denote the solution to this problem by $s^{A}(a)$. 


\section{B. Ex Post Utilitarianism}

For a given vector a,

(A. 3)

$$
\underset{s_{1}, s_{2}, \cdots s_{k}}{\operatorname{Max}} \frac{1}{i=1} \sum_{i}^{k} u\left(s_{i}, a_{i}\right)+w\left(m-\sum_{i=1}^{k} s_{i}\right) .
$$

Denote the solution to this problem by $s^{B}(a)$.

Theorem. If the probability function $F(a)$ is symmetric, i.e., $F(a)=$ $F\left(a^{\prime}\right)$, where $a^{\prime}$ is a permutation of $a$, then Problems $A$ and $B$ are equivalent, i.e., $s^{A}(a)=s^{B}(a)$ for all a.

Proof. The F.O.C. of Problem A are

$$
\begin{aligned}
& u_{1}\left(s_{1}(a), a_{1}\right)\left(1-\sum_{i=2}^{k} \lambda_{i}\right)-w^{\prime}\left(m-\sum_{i=1}^{k} s_{i}\right)=0, \\
& -w^{\prime}\left(m-\sum_{i=1}^{k} s_{i}\right)+\lambda_{i} u_{1}\left(s_{i}(a), a_{i}\right)=0, i=2,3, \cdots, k
\end{aligned}
$$

and (A.2), where $\lambda_{2}, \lambda_{3}, \cdots, \lambda_{k}$ are $k-1$ constants.

The F.O.C. for Problem B are

$$
\frac{1}{k} u_{1}\left(s_{i}, a_{i}\right)-w^{\prime}\left(m-\sum_{i=1}^{k} s_{i}\right)=0, i=1,2, \cdots, k \text {. }
$$

Set $\lambda_{i}=1 / k$ in $(A .4)$ and (A.5). Then the solution to (A.6) satisfies (A.4) and (A.5). We shall show that this solution also satisfies (A.2) and hence is a solution of Problem A.

Consider a solution to (A.6) associated with $a=\left(a_{1}, a_{2}, \cdots, a_{i}, \cdots, a_{k}\right)$ and a permutation $a^{\prime}=\left(a_{i}, a_{2}, \cdots, a_{1}, \cdots, a_{k}\right)$. Relabeling the solution correspondingly, the same set of equations is seen to be satisfied. That is, the following symmetry condition holds: 
(A.7)

$$
s_{1}\left(a_{1}, a_{2}, \cdots, a_{i}, \cdots, a_{k}\right)=s_{i}\left(a_{i}, a_{2}, \cdots, a_{1}, \cdots, a_{k}\right)
$$

Therefore,

$$
\begin{aligned}
& E\left[u\left(s_{i}(a), a_{i}\right)\right] \\
& =\iint \cdots \int u\left(s_{i}\left(a_{1}, a_{2}, \cdots, a_{i}, \cdots, a_{k}\right), a_{i}\right) d F\left(a_{1}, a_{2}, \cdots, a_{i}, \cdots, a_{k}\right) .
\end{aligned}
$$

Relabeling variables

$$
=\iint \cdots \int u\left(s_{i}\left(a_{i}, a_{2}, \cdots, a_{1}, \cdots, a_{k}\right), a_{1}\right) d F\left(a_{i}, a_{2}, \cdots, a_{1}, \cdots, a_{k}\right),
$$

by (A.7) and the symmetry of $F(a)$,

$$
\begin{aligned}
& =\iint \cdots \int u\left(s_{1}\left(a_{1}, a_{2}, \cdots, a_{i}, \cdots, a_{k}\right), a_{1}\right) d F\left(a_{1}, a_{2}, \cdots, a_{i}, \cdots, a_{k}\right) \\
& =E\left[u\left(s_{1}(a), a_{1}\right)\right] .
\end{aligned}
$$

thus satisfying (A.2).

Remark. The above theorem can be illustrated geometrically for a family with two children whose respective abilities assume the values $\left(a_{1}^{0}, a_{2}^{0}\right)$ and $\left(a_{2}^{0}, a_{1}^{0}\right)$ with equal probabilities. Due to symmetry, (A.7), the same total $s_{1}+s_{2}=\bar{s}$ will be distributed to the children in both cases. The efficient utility pairs $\left(u_{1}, u_{2}\right)$, given $\bar{s}$, corresponding to the two realizations are the curves $A A$ and $B B$ in Figure A.1. Pairs that yield equal expected utilities must be equally distant from the $45^{\circ}$ line. The pair that yields the highest equal levels of expected utilities is such that the line connecting them is bisected by the $45^{\circ}$ line and is tangent to both utility frontiers. Such a line exists because of symmetry of the utility frontiers. The implied allocations, $a$ on $A A$ and $b$ on $B B$, maximize the sum of utilities for each realization of the ability vector. 
A-4

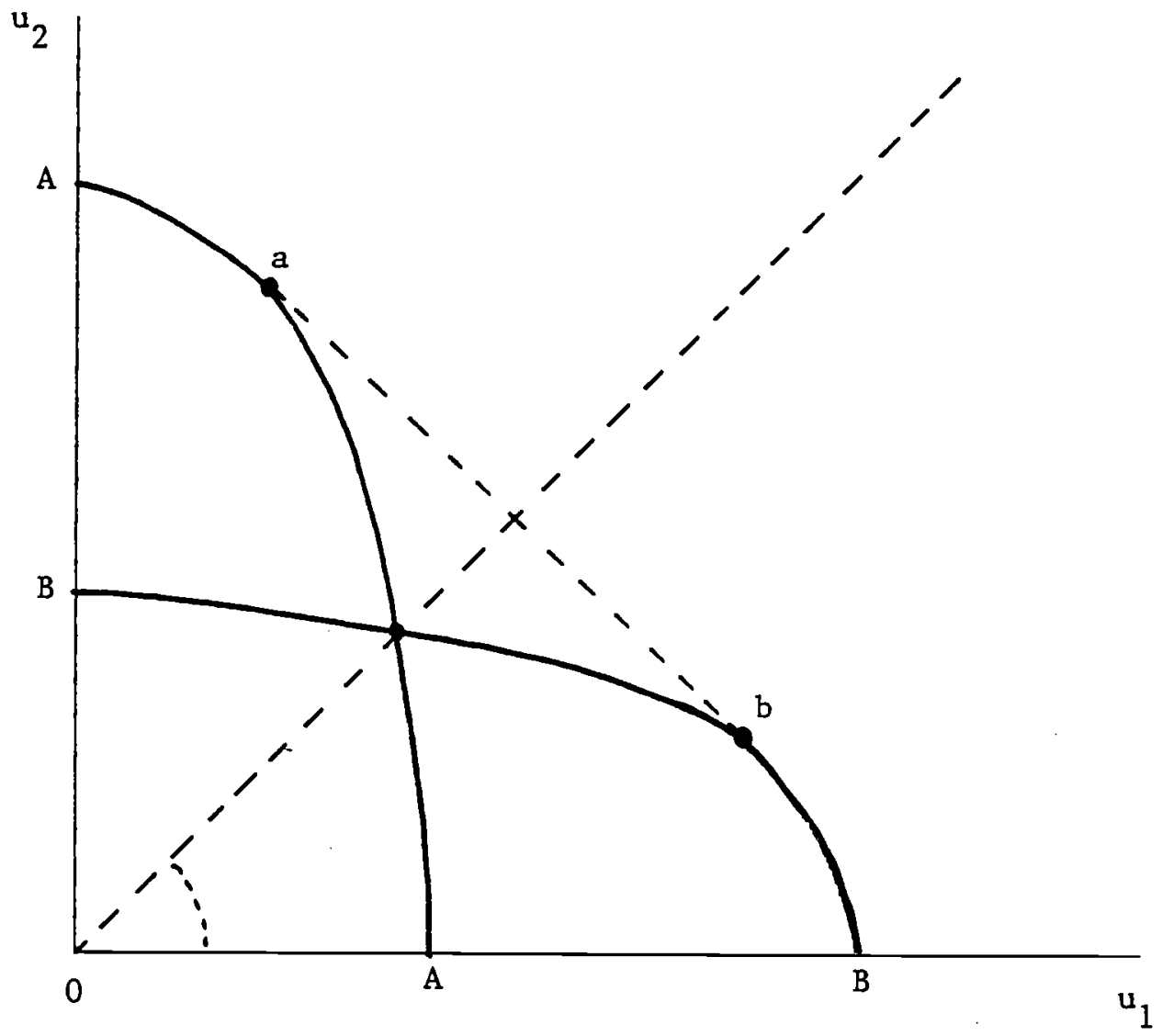

Figure A.1 